\title{
Polarisation measurement on three alternatives
}

Joost Veth*

\begin{abstract}
This bachelor thesis adresses the principle of polarisation on three different alternatives. It finds three different functions that find a measure for polarisation, that satisfy certain constraints. Then it argues about the usefulness of these functions.
\end{abstract}

\section{Introduction}

In this bachelor thesis, we introduce the topic of polarisation. Polarisation is a measurement to which extent different preferences can be harmonized. When there is low polarisation, it is easy to harmonize the different preferences, whereas this is difficult with high polarisation. There has been prior research in the field of polarisation (Esteban, J.-M. and Ray, D. (1994)., Duclos, J.-Y., Esteban, J., and Ray, D. (2004) and Baldiga, K. A. and Green, J. R. (2013)), but these studies were all on a one-dimensional scale (left/right, high/low), that is, people can only choose between two different alternatives. In this thesis we study polarisation measurement for arbitrary combinations of individual preferences, on three alternatives. Our goal is to find reasonable measures expressing polarisation. We take the normative way. So, these measures should satisfy certain desirable constraints/properties.

This thesis will be structured as follows: First we have a section where we describe the model, and explain the definitions we use and the constraints that we impose on the functions. Then, we introduce three different functions to measure polarisation on three alternatives, when considering two agents. We investigate whether these functions fulfill the constraints. Next, we investigate how the functions that do satisfy the constraints can be extended to more than 2 agents. Finally, we give some recommendations for further research.

\footnotetext{
Joost Veth graduated for his bachelor Econometrics and Operations Research at Maastricht University in July 2014, and is currently doing his master in Economic and Financial Research at Maastricht University.

Contact: J.veth@student . maastrichtuniversity.nl
} 


\section{Polarisation measurement on three alternatives}

\section{Model and Definitions}

Let $A=\left\{a_{1}, a_{2}, \ldots, a_{m}\right\}$ be the set of alternatives.

Let $N$ be the set of agents, that can indicate their preferences over $A$.

When an agent indicates his preference, for example agent i's top choice is alternative a, his second choice is alternative $\mathrm{b}$ and his third choice is alternative $\mathrm{c}$, we denote this as $(a, b, c)$. We call $(a, b, c)$ agent i's preferred order.

Preferences are formalised by Linear Orders. These are complete, antisymmetric and transitive relations on A. This defines the interpretation of a pair that is in the relation $S$.

When $a b \in S$ we say that a is weakly preferred to b. Furthermore, when $a b \in S$ and $b a \notin S$, we say that a is strictly preferred to $\mathrm{b}$.

A relation $S \subseteq A \times A$ is complete if $a b \in S$ or $b a \in S$, for all alternatives $a, b \in A$.

If $a b \in S$ implies $b a \notin S$ for all alternatives $a, b \in A$, with $a \neq b$, then the relation $S$ is antisymmetric;

When $a_{i} a_{j} \in S$ and $a_{j} a_{k} \in S$ implies that $a_{i} a_{k} \in S$ for all $i, j, k \in\{1, \ldots, m\}$, relation $S$ is transitive. In words: if $a_{i}$ is preferred over $a_{j}$, and $a_{j}$ is preferred over $a_{k}$, then $a_{i}$ is preferred over $a_{k}$.

Let $L$ denote the set of all linear orders on A.

It is possible to put different agents with possibly different preferred orders together in sets, $\left\{R_{1}, \ldots, R_{l}\right\}$.

For a set $R_{i}$, we define $D_{i}$ as the set of dominance pairs, a set with all the pairs $a b \in A \times A$ for which it holds that the first entrance of the pair is strictly preferred over the second entrance by at least one of the agents in that set.

For example, if agent $\mathrm{i}$ has preferred order $(a, b, c)$ and agent $\mathrm{j}$ has preferred order $(\mathrm{c}, \mathrm{a}, \mathrm{b})$, and they are together in set $R_{1}$, then $D_{1}=\{a b, a c, c a, b c, c b\}$.

Furthermore, the symmetric difference of two sets $D_{1}$ and $D_{2}, D_{1} \triangle D_{2}$ is the set of the elements that are in one of the sets, but not in their intersection.

So, if for example agent $\mathrm{i}$ has order $(\mathrm{a}, \mathrm{b}, \mathrm{c})$ and is in set $R_{1}$, and agent $\mathrm{j}$ has order $(\mathrm{c}, \mathrm{a}, \mathrm{b})$, and is in $R_{2}$, then $D_{1} \triangle D_{2}=\{a c, c a, b c, c b\}$.

The preference of agent $\mathrm{i}$ is denoted by $p(i)$.

A profile is a combination of linear orders: $p: N \rightarrow L$, where $N$ is the set of agents. $L^{N}$ denotes the set of profiles.

We define $\bar{p}_{a b}$ to be the number of times alternative $\mathrm{a}$ is preferred over alternative $\mathrm{b}$ at profile p. For $m$ alternatives, this gives for profile $\mathrm{p}$ an $m \times m$ matrix $\bar{P}$ of pairwise comparisons of the following form: 


\section{Polarisation measurement on three alternatives}

$$
\bar{P}=\left[\begin{array}{ccccc}
0 & \bar{p}_{a_{1} a_{2}} & \bar{p}_{a_{1} a_{3}} & \cdots & \bar{p}_{a_{1} a_{m}} \\
\bar{p}_{a_{2} a_{1}} & 0 & \bar{p}_{a_{2} a_{3}} & \cdots & \bar{p}_{a_{2} a_{m}} \\
\bar{p}_{a_{3} a_{1}} & \bar{p}_{a_{3} a_{2}} & 0 & \cdots & \bar{p}_{a_{3} a_{m}} \\
\vdots & \vdots & \vdots & \ddots & \vdots \\
\bar{p}_{a_{m} a_{1}} & \bar{p}_{a_{m} a_{2}} & \bar{p}_{a_{m} a_{3}} & \cdots & 0
\end{array}\right]
$$

Note: for a profile $\mathrm{q}, \bar{Q}$ is its matrix of pairwise comparisons.

Define $\hat{p}_{a b}=\bar{p}_{a b}-\bar{p}_{b a}$, or, in words, the net preference between a and $\mathrm{b}$.

We say that profiles $p$ and $q$ are pairwise conflict free, if the following holds: For all alternatives $a_{i} a_{j} \in A \times A,(i, j \in 1, \ldots, m)$, if $\hat{p}_{a b}>0$, then $\hat{q}_{a b} \geq 0$ and $\hat{q}_{a b}>0$ implies that $\hat{p}_{a b} \geq 0$.

Now, we introduce a function, $\phi: L^{n} \rightarrow R^{+}$, to measure polarisation.

Furthermore, we state a couple of constraints on the function $\phi$ :

1) Regularity: 1.1: $\phi\left(R^{n}\right)=0$, where $R^{n}=(R, R, \ldots, R)$ is a profile where all the linear orders are the same;

1.2: $\phi\left(R^{N_{1}},(-R)^{N_{2}}\right)=1$, whenever the number of elements in $N_{1}$ is equal to the number of elements in $N_{2}$.

2) Neutrality : $\phi(p)=\phi(\pi p)$ for any permutation $\pi: A \times A$, where $\pi p(i)=\{\pi(x) \pi(y)$ : $x y \in p(i)\}$ and $\pi p=(\pi p(1), \pi p(2), \ldots, \pi p(n))$. In words, this means that the names of the alternatives have no influence on the measure of polarisation. For example, if $R_{1}=(a, b, c)$, $R_{2}=(a, c, b), R_{3}=(c, a, b)$ and $R_{4}=(c, b, a)$, and $\pi(a)=c, \pi(b)=a, \pi(c)=b$, then neutrality means that $\phi\left(R_{1}, R_{2}\right)=\phi\left(R_{3}, R_{4}\right)$.

3) Pairwiseness : if for profile $\mathrm{p}$ and $\mathrm{q}, \bar{P}=\bar{Q}$, then $\phi(p)=\phi(q)$. In words: if more than one profile leads to the same matrix of pairwise comparisons, then these profiles have the same measure of polarisation.

4) Additivity : If profile $p$ and $q$ are pairwise conflict free, then it holds that $\frac{n}{n+m} \phi(p)+$ $\frac{m}{n+m} \phi(q)=\phi(p, q)$ if there are $n$ agents in $p$ and $m$ agents in $q$.

We start by considering the following situation: We have two agents, say agent $\mathrm{i}$ and $\mathrm{j}$, and 3 alternatives, called a,b and c.

These two agents indicate their preferences about the 3 alternatives.

Once they have chosen their preference, we are interested in the measure of polarisation of those two preferences. The situation is illustrated in figure 1. 


\section{Polarisation measurement on three alternatives}

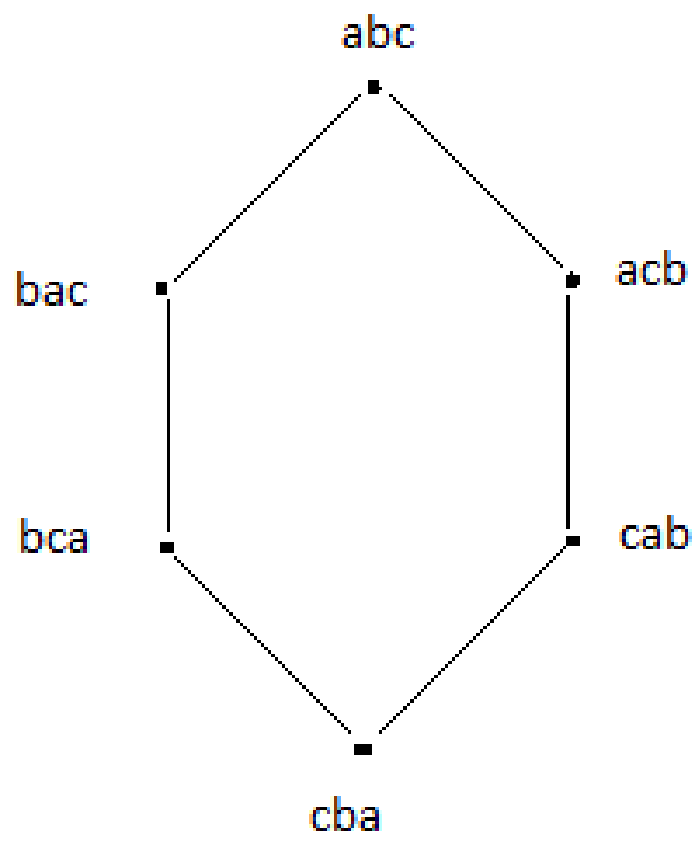

Figure 1 - Graphical illustration of the situation with three alternatives.

\section{Functions}

In this section, we will introduce three different functions that measure polarisation on three alternatives, and we investigate whether these functions satisfy the different constraints for the case with two agents.

\subsection{Function 1}

The method we are going to use is to start with a function that satisfies the regularity constraint, and then see if this function also satisfies the other constraints. If so, we try to extend this function to more than two agents. If this function does not satisfy all the constraints, we will search for other functions that do satisfy all the constraints.

So, first we try to find a function $\phi$ that satisfies the constraint of regularity in the case of two agents, $\mathrm{i}$ and $\mathrm{j}$.

In this approach, we allocate three different scores to the preferences of agent $\mathrm{i}$ and $\mathrm{j}$. These score are 1,0 and -1 .

Let $R_{1}=a_{1}, a_{2}, a_{3}$ and $R_{2}=a_{j 1}, a_{j 2}, a_{j 3}$. That is, the top choice of agent 1 is $a_{1}$, his second choice $a_{2}$ and his third choice $a_{3}$, and the top choice of agent 2 is $a_{j 1}$, his second choice $a_{j 2}$ and 


\section{Polarisation measurement on three alternatives}

his third choice $a_{j 3}$.

Then, we allocate the scores in the following way:

$$
\begin{gathered}
\operatorname{score}\left(1, a_{t}\right)= \begin{cases}1 & \text { if } t=1 \\
0 & \text { if } t=2 \\
-1 & \text { if } t=3\end{cases} \\
\operatorname{score}\left(2, a_{j t}\right)= \begin{cases}1 & \text { if } t=a_{1} \\
0 & \text { if } t=a_{2} \\
-1 & \text { if } t=a_{3}\end{cases}
\end{gathered}
$$

So for example, if agent i's preferred order is $(a, b, c)$, then $a_{1}$ gets score $1, a_{2}$ gets score 0 and $a_{3}$ gets score -1 . Then, if the preferred order of agent $\mathrm{j}$ is $(b, c, a)$, then $a_{j 1}$ receives score 0 , $a_{j 2}$ receives score -1 and $a_{j 3}$ receives score 1 .

The following function gives a polarisation measure to all possible combinations of preferences for agent $i$ and $j$ that satisfy the regularity constraint:

$$
\text { (1) } \phi\left(R_{1}, R_{2}\right)=\frac{-\left(\sum_{t=1}^{3}\left(\operatorname{score}\left(1, a_{t}\right) \times \operatorname{score}\left(2, a_{j t}\right)\right)\right)+2}{4}
$$

Now, there are four possible outcomes:

1) Agent $\mathrm{i}$ and agent $\mathrm{j}$ both have as preferred order $(\mathrm{a}, \mathrm{b}, \mathrm{c})$. Then $\left(\sum_{t=1}^{3}\left(\operatorname{score}\left(1, a_{t}\right) \times\right.\right.$ $\left.\left.\operatorname{score}\left(2, a_{j t}\right)\right)\right)=2$, and $-\left(\sum_{t=1}^{3}\left(\operatorname{score}\left(1, a_{t}\right) \times \operatorname{score}\left(2, a_{j t}\right)\right)\right)+2=0$. Hence $\phi=0$.

2) Agent $i$ has as preferred order $(a, b, c)$ and agent $j$ has as preferred order either $(a, c, b)$ or $(\mathrm{b}, \mathrm{a}, \mathrm{c})$. In these cases, $\left(\sum_{t=1}^{3}\left(\operatorname{score}\left(1, a_{t}\right) \times \operatorname{score}\left(2, a_{j t}\right)\right)\right)=1$ and $-\left(\sum_{t=1}^{3}\left(\operatorname{score}\left(1, a_{t}\right) \times\right.\right.$ $\left.\left.\operatorname{score}\left(2, a_{j t}\right)\right)\right)+2=1$. Hence, $\phi=\frac{1}{4}$.

3) Agent $i$ has as preferred order $(a, b, c)$ and agent $j$ has as preferred order either $(c, a, b)$ or $(\mathrm{b}, \mathrm{a}, \mathrm{c})$. Then, $\left(\sum_{t=1}^{3}\left(\operatorname{score}\left(1, a_{t}\right) \times \operatorname{score}\left(2, a_{j t}\right)\right)\right)=-1$, and $-\left(\sum_{t=1}^{3}\left(\operatorname{score}\left(1, a_{t}\right) \times \operatorname{score}\left(2, a_{j t}\right)\right)\right)+$ $2=3$. Hence, $\phi=\frac{3}{4}$.

4) Agent $i$ has as preferred order $(a, b, c)$ and agent $j$ has as preferred order $(c, b, a)$. Then $\left(\sum_{t=1}^{3}\left(\operatorname{score}\left(1, a_{t}\right) \times \operatorname{score}\left(2, a_{j t}\right)\right)\right)=-2$, and $-\left(\sum_{t=1}^{3}\left(\operatorname{score}\left(1, a_{t}\right) \times \operatorname{score}\left(2, a_{j t}\right)\right)\right)+2=4$. Thus $\phi=1$.

For every other order agent i can choose, an analogous analysis can be applied.

This function satisfies the regularity constraint.

Moreover, it satisfies the neutrality constraint. This is guaranteed by the fact that the values for $a_{1}, a_{2}$ and $a_{3}$ are always 1,0 and -1 respectively, independent of the preference of agent $\mathrm{i}$. 


\section{Polarisation measurement on three alternatives}

Hence, as long as the distance between the two agents is the same, the function will give the same score for the polarisation. Thus, neutrality is satisfied.

Pairwiseness is also satisfied. To prove that the constraint of pairwiseness is also satisfied, we consider all the possible cases and we prove for each individual case that pairwiseness is satisfied.

Without loss of generality, we assume that the preferred order of agent $i$ is $(a, b, c)$.

Case 1: The preferred order of agent $j$ is also $(a, b, c)$. Then by the regularity constraint, the polarisation measure is always 0 . Thus, pairwiseness is satisfied;

Case 2: The preferred order of agent $\mathrm{j}$ is $(\mathrm{a}, \mathrm{c}, \mathrm{b})$. Then, $\bar{P}_{2}=\left[\begin{array}{lll}0 & 2 & 2 \\ 0 & 0 & 1 \\ 0 & 1 & 0\end{array}\right]$. When you consider

$\bar{P}_{2}$, we see that for both agents, $a$ is preferred over $\mathrm{b}$, and $\mathrm{a}$ is preferred over $\mathrm{c}$. So, for both agents, $a$ is the first choice. Furthermore, one agent prefers $b$ over $c$, and the other prefers $c$ over b. Since we assumed that the preference of agent $i$ is $(a, b, c)$, and thus that agent i prefers b over $\mathrm{c}$, there is no other way to get $\bar{P}_{2}$ other than that agent j prefers $\mathrm{c}$ over $\mathrm{b}$, and thereby has as preferred order $(a, c, b)$. Since $\bar{P}_{2}$ can only be reached in one way, pairwiseness is satisfied.

Case 3: The preferred order of agent $\mathrm{j}$ is $(\mathrm{c}, \mathrm{a}, \mathrm{b})$. Then, $\bar{P}_{3}=\left[\begin{array}{ccc}0 & 2 & 1 \\ 0 & 0 & 1 \\ 1 & 1 & 0\end{array}\right]$. When you consider $\bar{P}_{3}$, we see that c is preferred over a by one agent, and the other prefers a over c. Since agent $\mathrm{i}$ prefers a over c, it must always be the case that agent j prefers c over a. Similarly, one agent prefers $c$ over $b$ and the other way around, and since agent i prefers $b$ over $c$, agent $j$ must prefer $c$ over $b$. Furthermore, they both prefer a over $b$. Altogether, there is only one option for the order of agent $\mathrm{j}:(\mathrm{c}, \mathrm{a}, \mathrm{b})$. Again, pairwiseness is satisfied.

Case 4: The preferred order of agent $\mathrm{j}$ is $(\mathrm{c}, \mathrm{b}, \mathrm{a})$. Then by the regularity condition, the polarisation measure is always 1 . Thus, pairwiseness is satisfied;

Since these are all the possible scenarios, we can conclude that pairwiseness is satisfied.

However, this function does not satisfy the additivity constraint.

proof: To prove that this function does not satisfy additivity, we have to consider three different agents, since additivity has no bite in case there are at most 2 agent (which will be showed later on). So we consider three agents, 1,2 and 3. Agent 1 has as preferred order $R_{1}: p(1)=(a, b, c)$, for agent 2, $R_{2}: p(2)=(a, c, b)$ and for agent 3, $R_{3}: p(3)=(c, a, b)$. Then $\phi\left(R_{1}, R_{2}\right)=\frac{1}{4}, \phi\left(R_{2}, R_{3}\right)=\frac{1}{4}$ and $\phi\left(R_{1}, R_{3}\right)=\frac{3}{4}$. Their respective matrices of pairwise 


\section{Polarisation measurement on three alternatives}

comparisons are:

$\bar{P}_{12}=\left[\begin{array}{lll}0 & 2 & 2 \\ 0 & 0 & 1 \\ 0 & 1 & 0\end{array}\right], \bar{P}_{23}=\left[\begin{array}{lll}0 & 2 & 1 \\ 0 & 0 & 0 \\ 1 & 2 & 0\end{array}\right]$ and $\bar{P}_{13}=\left[\begin{array}{ccc}0 & 2 & 1 \\ 0 & 0 & 1 \\ 1 & 1 & 0\end{array}\right]$. Since these matrices are all pairwise conflict free, $\phi\left(R_{1}, R_{2}, R_{2}, R_{3}\right)=\frac{1}{2} \phi\left(R_{1}, R_{2}\right)+\frac{1}{2} \phi\left(R_{2}, R_{3}\right)$ according to the additivity constraint. Hence, $\phi\left(R_{1}, R_{2}, R_{2}, R_{3}\right)=\frac{1}{2} \times \frac{1}{4}+\frac{1}{2} \times \frac{1}{4}=\frac{1}{4}$. But also, $\phi\left(R_{1}, R_{2}, R_{2}, R_{3}\right)=$ $\frac{1}{2} \phi\left(R_{2}, R_{2}\right)+\frac{1}{2} \phi\left(R_{1}, R_{3}\right)$, thus $\phi\left(R_{1}, R_{2}, R_{2}, R_{3}\right)=\frac{1}{2} \times 0+\frac{1}{2} \times \frac{3}{4}=\frac{3}{8}$. This proves that the additivity constraint is not satisfied.

\subsection{Function 2}

In this approach, we take a look at the situation with two agents and three alternatives from a different point of view. When we compare two different preferences, we take a look at the number of times that two adjacent elements have to be swapped to go from one order to the other. For example, to go from $(a, b, c)$ to $(c, a, b)$, two swaps have to be made.

Now, we try to allocate a weight to the conflict that arises when a swap has to be made to unite two different preferences, called the weight of a swap, $w\left(a_{1} a_{2}\right)$. Then, the polarity measure of two preferences is defined as the sum of the weights of all the swaps that have to be made to unite the two preferences.

Preferably, we distinguish between a 'high value' swap ( when we do $(a, b, c,) \rightarrow(b, a, c)$, the top two alternatives are swapped) and a 'low value' $\operatorname{swap}((a, b, c) \rightarrow(a, c, b))$. The idea behind this, is that people often value their first preference higher than their second and third preference. That is, if they prefer the order $(a, b, c)$, they will feel harmed more when the order $(b, a, c)$ is chosen, than when the order $(a, c, b)$ is chosen. Optimally, this would be reflected in the measure of polarisation. Unfortunately, this property is in conflict with the additivity constraint.

proof We prove this by contradiction:

Assume we can distinguish between a 'low value' swap $w_{l}\left(a_{1} a_{2}\right)$, and a 'high value' swap, $w_{h}\left(a_{1} a_{2}\right)$ :

To go from (a,b,c) to (c,b,a) takes three swaps: either (a,b,c) $\rightarrow(b, a, c) \rightarrow(b, c, a) \rightarrow(c, b, a)$, call this path 1 , or $(a, b, c) \rightarrow(a, c, b) \rightarrow(c, a, b) \rightarrow(c, b, a)$, path 2 . First, we look at path 1 . We defined $w\left(a_{1} a_{2}\right)$ as 'the conflict that arises when there has to be made a swap', and when the swap is made between the two first elements, we say this is a 'high value' swap, otherwise we have a 'low value' swap. So, the conflict that arises by the swap from $(\mathrm{a}, \mathrm{b}, \mathrm{c})$ to $(\mathrm{b}, \mathrm{a}, \mathrm{c})$ is $w_{h}(a b)$. For the swap from $(\mathbf{b}, \mathbf{a}, \mathbf{c})$ to $(\mathbf{b}, \mathbf{c}, \mathbf{a})$, this is $w_{l}(a c)$ and for the swap from $(\mathbf{b}, \mathbf{c}, \mathbf{a})$ to $(\mathbf{c}, \mathbf{b}, \mathbf{a})$ this is $w_{h}(b c)$. 


\section{Polarisation measurement on three alternatives}

Now consider 4 agents: Agent 1 has as preferred order $R_{1}: p(1)=(a, b, c)$, agent 2 has $R_{2}$ : $p(2)=(b, a, c)$, agent 3 has $R_{3}: p(3)=(b, c, a)$ and agent 4 has $R_{4}: p(4)=(c, b, a)$.

Then $\bar{P}_{12}=\left[\begin{array}{lll}0 & 1 & 2 \\ 1 & 0 & 2 \\ 0 & 0 & 0\end{array}\right], \bar{P}_{24}=\left[\begin{array}{lll}0 & 0 & 1 \\ 2 & 0 & 1 \\ 1 & 1 & 0\end{array}\right]$ and $\bar{P}_{14}=\left[\begin{array}{ccc}0 & 1 & 1 \\ 1 & 0 & 1 \\ 1 & 1 & 0\end{array}\right]$. Since these matrices are all pairwise conflict free, $\phi\left(R_{1}, R_{2}, R_{2}, R_{4}\right)=\frac{1}{2} \phi\left(R_{1}, R_{2}\right)+\frac{1}{2} \phi\left(R_{2}, R_{4}\right)$ according to the additivity constraint.

But also $\phi\left(R_{1}, R_{2}, R_{2}, R_{4}\right)=\frac{1}{2} \phi\left(R_{1}, R_{4}\right)+\frac{1}{2} \phi\left(R_{2}, R_{2}\right)$. Since $\phi\left(R_{2}, R_{2}\right)=0$, we know that $\frac{1}{2} \phi\left(R_{1}, R_{4}\right)=\frac{1}{2} \phi\left(R_{1}, R_{2}\right)+\frac{1}{2} \phi\left(R_{2}, R_{4}\right)$. Hence, $\phi\left(R_{1}, R_{4}\right)=\phi\left(R_{1}, R_{2}\right)+\phi\left(R_{2}, R_{4}\right)(\star)$.

Moreover, $\bar{P}_{23}=\left[\begin{array}{lll}0 & 1 & 1 \\ 1 & 0 & 2 \\ 1 & 0 & 0\end{array}\right]$ and $\bar{P}_{34}=\left[\begin{array}{lll}0 & 0 & 0 \\ 2 & 0 & 1 \\ 2 & 1 & 0\end{array}\right]$. These matrices are pairwise conflict free as well. Therefore, again by the additivity constraint, $\phi\left(R_{2}, R_{3}, R_{3}, R_{4}\right)=\frac{1}{2} \phi\left(R_{2}, R_{3}\right)+$ $\frac{1}{2} \phi\left(R_{3}, R_{4}\right)$, and $\phi\left(R_{2}, R_{3}, R_{3}, R_{4}\right)=\frac{1}{2} \phi\left(R_{2}, R_{4}\right)+\frac{1}{2} \phi\left(R_{3}, R_{3}\right)$. Hence, $\phi\left(R_{2}, R_{4}\right)=\phi\left(R_{2}, R_{3}\right)+$ $\phi\left(R_{3}, R_{4}\right)(\star \star)$.

From $(\star)$ and $(\star \star)$, it follows that $\phi\left(R_{1}, R_{4}\right)=\phi\left(R_{1}, R_{2}\right)+\phi\left(R_{2}, R_{3}\right)+\phi\left(R_{3}, R_{4}\right)$. To go from $R_{1}$ to $R_{2}$, you swap from (a,b,c) to (b,a,c), so the conflict that arises by the swap is $w_{h}(a b)$. Thus, $\phi\left(R_{1}, R_{2}\right)=w_{h}(a b)$. Similarly, $\phi\left(R_{2}, R_{3}\right)=w_{l}(a c)$ and $\phi\left(R_{3}, R_{4}\right)=w_{h}(b c)$. By the regularity constraint, we know that $\phi((a, b, c),(a, b, c))=0$, and $\phi((a, b, c),(c, b, a))=1$.

Thus, $w_{h}(a b)+w_{l}(a c)+w_{h}(b c)=1$.

For path 2, we find by the same reasoning that $w_{l}(b c)+w_{h}(a c)+w_{l}(a b)=1$.

Therefore, $w_{h}(a b)+w_{l}(a c)+w_{h}(b c)=w_{l}(b c)+w_{h}(a c)+w_{l}(a b)$.

In an analogous way, one can find that $w_{l}(a c)+w_{h}(a b)+w_{l}(b c)=w_{h}(b c)+w_{l}(a b)+w_{h}(a c)$, and they also both add up to 1 . But this would mean that

$w_{l}(b c)+w_{h}(a c)+w_{l}(a b)=w_{h}(b c)+w_{l}(a b)+w_{h}(a c)$. Hence, that $w_{l}(b c)=w_{h}(b c)$. A similar analysis could be done on all other possible pairs, contradicting our assumption that we can distinguish between a 'low value' swap $w_{l}\left(a_{1} a_{2}\right)$, and a 'high value' swap, $w_{h}\left(a_{1} a_{2}\right)$. This proves that we cannot distinguish between 'high' and 'low' values.

In this function, we make use of the concept of the symmetric difference of two sets $D_{1}$ and $D_{2}, D_{1} \triangle D_{2}$, which we defined as the set of the elements that are in one of the sets, but not in their intersection.

The two sets $D_{1}$ and $D_{2}$ are both a set of dominance pairs, (in the set are all the pairs for which it holds that the first entrance of the pair is strictly preferred over the second entrance by 


\section{Polarisation measurement on three alternatives}

at least one of the agents in the set) for two different profiles.

If for example $R_{1}$ consists of only agent $\mathrm{i}$, which has as order (a,b,c) and $R_{2}$ consists of only agent $\mathrm{j}$, with order (b,c,a), then $D_{1} \triangle D_{2}=\{a b, a c, b a, c a\}$.

Note that in this symmetric difference, each pair that has to be swapped to go from the first order $(a, b, c)$ to the second $(b, c, a)$, namely $a b$ and ac, is present twice.

It can be shown that this is always the case.

Taking this as a starting point, We take all the elements in the symmetric difference of $\mathrm{n}$ orders, then we take the sum of their respective weights, and then we divide this number by the number of agents, $\mathrm{n}$, to get a value for the polarisation function $\phi$ :

$$
\text { (2) } \phi\left(R_{1}, R_{2}\right)=\frac{\sum_{x y \in D_{1} \triangle D_{2}} w(x, y)}{n}
$$

Since $\phi((a, b, c),(c, b, a))=1$, we know that $\frac{2 w(a b)+2 w(a c)+2 w(b c)}{2}=1$. Hence, $2 w(a b)+2 w(a c)+$ $2 w(b c)=2$, therefore $w(a b)+w(a c)+w(b c)=1$.

For simplicity, we now assume that the polarisation is spread equally over the different pairs, thus $w(a b)=w(a c)=w(b c)=\frac{1}{3}$.

If this is the case,

Regularity is satisfied, since when the two pairs are equal, then $D_{1} \triangle D_{2}=\emptyset$, thus the numerator of $\phi\left(R_{1}, R_{2}\right)=0$, hence $\phi\left(R_{1}, R_{2}\right)=0$, and we already have seen that $\phi\left(R^{N 1},(-R)^{N 2}\right)=1$.

Neutrality is only satisfied if all the different weights are valued equally. Since this is the case, Neutrality is satisfied;

As proved before, pairwiseness is always satisfied for two agents.

In the case of two agents, these agents can either have the same preference, or a different preference. In the case that the two agents ( $i$ and $j$ ) have the same preference, $\phi(i, j)=0$. Since $\phi(i)=\phi(j)=0$ by definition, since there is no polarisation when only one agent indicated his preference, it is automatically true that $\phi(i, j)=\frac{1}{2} \phi(i)+\frac{1}{2} \phi(j)$. Thus, when the two agents have the same preference, additivity automatically follows from regularity.

When the two agents don't have the same preference, their respective matrices of pairwise comparisons are conflicting for at least one pair of preferences $i, j \in A$. Therefore, additivity does not apply in these cases. Hence, the additivity rule is always satisfied when you only consider 2 agents. Thus, this function satisfies all the constraints for two agents.

From three agents on, this approach doesn't work anymore, and we have to adapt our function. This will be done in section 4 . 


\section{Polarisation measurement on three alternatives}

\subsection{Function 3}

We also considered a third function to measure polarisation. This function is inspired by the principle of the Cobb-Douglas utility function [4], where the maximum consumer utility is calculated in a similar way as the denominator of this function is calculated.

In this approach, the matrix of pairwise comparisons plays a central role. Each profile has such a matrix. In this approach, we multiply each entry $\bar{p}_{a b},(a \neq b)$ of this matrix that is in the upper triangular (that is, each entry that is above the diagonal) by its reversed entry in the lower triangular. Then we sum up all these products, and divide this by three times the maximum value of such a product. If $n$, the number of agents, is odd, this maximum value is $\left(\frac{1}{2} n-\frac{1}{2}\right)\left(\frac{1}{2} n+\frac{1}{2}\right)$. If $\mathrm{n}$ is even, this maximum value is $\left(\frac{1}{2} n\right)^{2}$. More formally:

$$
\text { (3) } \phi= \begin{cases}\frac{\sum_{\forall 1 \leq i<j \leq m}\left(\bar{p}_{a b} \times \bar{p}_{b a}\right)}{3 \times\left(\frac{1}{2} n\right)^{2}} & (a \neq b), \text { if nis even } \\ \frac{\sum_{\forall 1 \leq i \leq m \leq m}\left(\bar{p}_{a b} \times \bar{p}_{b a)}\right.}{\frac{3}{4}\left(n^{2}-1\right)} & (a \neq b), \text { if } n \text { is odd }\end{cases}
$$

For two agents, agent $\mathrm{i}$ and $\mathrm{j}$, this function has four possible outcomes. We assume, without loss of generality, that agent $i$ has as preferred order $(a, b, c)$.

If agent $\mathrm{j}$ has preferred order $(\mathrm{a}, \mathrm{b}, \mathrm{c})$, we get as matrix of pairwise comparisons $\bar{P}_{1}=$ $\left[\begin{array}{lll}0 & 2 & 2 \\ 0 & 0 & 2 \\ 0 & 0 & 0\end{array}\right] ;$ In this case, $\phi=0$.

If agent $\mathrm{j}$ has preferred order $(\mathrm{a}, \mathrm{c}, \mathrm{b}), \bar{P}_{2}=\left[\begin{array}{lll}0 & 2 & 2 \\ 0 & 0 & 1 \\ 0 & 1 & 0\end{array}\right]$, and if agent $\mathrm{j}$ has preferred order (b,a,c), $\bar{P}_{6}=\left[\begin{array}{lll}0 & 1 & 2 \\ 1 & 0 & 2 \\ 0 & 0 & 0\end{array}\right]$. In both cases, $\phi=\frac{1}{3}$.

If agent $\mathrm{j}$ has as order $(\mathrm{c}, \mathrm{a}, \mathrm{b}), \bar{P}_{3}=\left[\begin{array}{lll}0 & 2 & 1 \\ 0 & 0 & 1 \\ 1 & 1 & 0\end{array}\right]$; If agent $\mathrm{j}$ has as order $(\mathrm{b}, \mathrm{c}, \mathrm{a})$, then 


\section{Polarisation measurement on three alternatives}

$\bar{P}_{5}=\left[\begin{array}{ccc}0 & 1 & 1 \\ 1 & 0 & 2 \\ 1 & 0 & 0\end{array}\right]$. In these cases, $\phi=\frac{2}{3}$

If agent $\mathrm{j}$ has as order $(\mathrm{c}, \mathrm{b}, \mathrm{a}), \bar{P}_{4}=\left[\begin{array}{lll}0 & 1 & 1 \\ 1 & 0 & 1 \\ 1 & 1 & 0\end{array}\right]$, and $\phi=1$.

The same analysis can be done for all other preferences agent i can choose.

Since the outcomes for $\phi$ for the different combinations of preferences of the two agents are all the same as they are for function two, we know that regularity, additivity, pairwiseness and neutrality are all satisfied.

\section{Extending to more than 2 agents}

\subsection{Function 2}

Our current function 2, defined in section 3.2, does not work for more than two agents.

proof Consider for example two situations where you have 2 sets, with two agents is both sets.

In situation 1, both agents 1 and 2 are in set $R_{1}$ and have preferred order (a,b,c). Moreover, both agents 3 and 4 are in set $R_{2}$ and have preferred order (c,b,a).

Then $D_{1}=\{\mathrm{ab}, \mathrm{ac}, \mathrm{bc}\}, D_{2}=\{\mathrm{ba}, \mathrm{ca}, \mathrm{cb}\}$ and $D_{1} \triangle D_{2}=\{a b, a c, b c, b a, c a, c b\}$. Then $\phi\left(R_{1}, R_{2}\right)=$ $\frac{1}{2}$.

In situation 2, the agents have all the same preferences (thus, the measure of polarisation should be the same), but now agent 1 and 3 are in set $R_{1}$, and agent 2 and 4 are in set $R_{2}$.

Then $D_{1}=\{\mathrm{ab}, \mathrm{ac}, \mathrm{bc}, \mathrm{ba}, \mathrm{ca}, \mathrm{cb}\}$ and $D_{2}=\{\mathrm{ab}, \mathrm{ac}, \mathrm{bc}, \mathrm{ba}, \mathrm{ca}, \mathrm{cb}\}$. Then $D_{1} \triangle D_{2}=\{\emptyset\}$. Hence $\phi\left(R_{1}, R_{2}\right)=0$. This cannot be the case.

The easiest way to tackle this problem is to reformulate the formula such that the agents are not divided in two groups, but that they are all considered together. We came up with the next function:

$$
\text { (4) } \phi(p)=\sum_{a b \in A \times A} \frac{w(a b) \times \min \left\{\bar{P}_{a b}, \bar{P}_{b a}\right\}}{n}
$$

For two agents, this formula gives the same result as (2): 


\section{Polarisation measurement on three alternatives}

$\min \left\{\bar{P}_{a b}, \bar{P}_{b a}\right\}$ can adopt two values, zero and one.

For a particular pair ab, if both agents prefer a over b, $\bar{P}_{a b}=2$ and $\bar{P}_{b a}=0$. In this case. $\min \left\{\bar{P}_{a b}, \bar{P}_{b a}\right\}=0$, and thus $w(a b)$ is not counted. For (2), in the same situation the pair ab would be in both sets $R_{1}$ and $R_{2}$, and therefore not be counted either.

For a pair ab, if one agent prefers a over b, and the other prefers b over a, $\bar{P}_{a b}=1$ and $\bar{P}_{b a}=1$. In this case, $\min \left\{\bar{P}_{a b}, \bar{P}_{b a}\right\}=1$, and thus $w(a b)$ is counted once. Note: $w(a b)$ is also counted when considering pair ba. Hence, every time that an alternative a is once preferred over another alternative $\mathrm{b}$, and $\mathrm{b}$ once preferred over $\mathrm{a}, w(a b)$ is counted twice. This is exactly what happens for (2) when such a situation occurs, since the two different pairs are in the different sets, but not in their intersection. This means that for each situation with two agents and three alternatives, (4) will generate the same outcome as (2).

For more than two agents, the function satisfies all the constraints:

When all the agents have the same preferences, $\min \left\{\bar{P}_{a b}, \bar{P}_{b a}\right\}=0$ for all pairs ab. This means that $\phi=0$. When half of the agents are in set $R_{1}$ and have the exact opposite preferred order compared to the other half of the agents in $R_{2}, \min \left\{\bar{P}_{a b}, \bar{P}_{b a}\right\}=\frac{1}{2 n}$ for all pairs ab, resulting in $\phi=1$. Hence, the regularity constraint is satisfied. Note that for an odd number of agents, it is not possible to obtain the value of 1 for $\phi$. But according to the regularity constraint, $\phi$ has to be equal to one only if the two sets $R_{1}$ and $R_{2}$ have the same amount of agents. This cannot be the case with an odd number of agents, therefore $\phi$ doesn't have to be able to receive the value of 1 in these cases.

Neutrality is also satisfied, since the different weights are valued equally;

pairwiseness is satisfied if different profiles that lead to the same matrix of pairwise comparisons, have the same measure of polarisation. In this function, when two profiles lead to the same matrix of pairwise comparisons, all the $\min \left\{\bar{P}_{a b}, \bar{P}_{b a}\right\}$ are the same for the two profiles, and therefore $\phi$ is equal for both profiles. Hence, pairwiseness is satisfied.

For additivity to be applicable at two profiles, $p$ and $q$, it must hold for all pairs ab, that if $\hat{p}_{a b}>0$, then $\hat{q}_{a b} \geq 0$ and $\hat{q}_{a b}>0$ implies that $\hat{p}_{a b} \geq 0$. In this function, this means that $\min \left\{\bar{Q}_{a b}, \bar{Q}_{b a}\right\}+\min \left\{\bar{P}_{a b}, \bar{P}_{b a}\right\}=\min \left\{\bar{Q}_{a b}+\bar{P}_{a b}, \bar{Q}_{b a}+\bar{P}_{b a}\right\}(\star)$.

This means that, when there are $\mathrm{n}$ agents in $\mathrm{p}$, and $\mathrm{m}$ agents in $\mathrm{q}$, and $\mathrm{p}$ and $\mathrm{q}$ are pairwise conflict free, then:

$$
\phi(p, q)=\sum_{a b \in A \times A} \frac{w(a b) \times \min \left\{\bar{Q}_{a b}+\bar{P}_{a b}, \bar{Q}_{b a}+\bar{P}_{b a}\right\}}{m+n}
$$

This is in correspondence with the additivity constraint: 


\section{Polarisation measurement on three alternatives}

proof: Consider two profiles, $\mathrm{p}$ and $\mathrm{q}$, that are both pairwise conflict free, and there are $\mathrm{n}$ agents in profile $p$, and $m$ agents in profile $q$.

Then according to the additivity constraint, the following must hold: $\frac{n}{n+m} \phi(p)+\frac{m}{n+m} \phi(q)=$ $\phi(p, q)$.

For this function, $\frac{n}{n+m} \phi(p)=\frac{n}{n+m} \sum_{a b \in A \times A} \frac{w(a b) \times \min \left\{\bar{P}_{a b}, \bar{P}_{b a}\right\}}{n}=\frac{n}{n(n+m)} \sum_{a b \in A \times A} w(a b) \times \min \left\{\bar{P}_{a b}, \bar{P}_{b a}\right\}$ $=\frac{\sum_{a b \in A \times A} w(a b) \times \min \left\{\bar{P}_{a b}, \bar{P}_{b a}\right\}}{n+m}$ and $\frac{m}{n+m} \phi(q)=\frac{m}{n+m} \sum_{a b \in A \times A} \frac{w(a b) \times \min \left\{\bar{Q}_{a b}, \bar{Q}_{b a}\right\}}{m}=\frac{m}{m(n+m)} \sum_{a b \in A \times A} w(a b) \times$ $\min \left\{\bar{Q}_{a b}, \bar{Q}_{b a}\right\}=\frac{\sum_{a b \in A \times A} w(a b) \times \min \left\{\bar{Q}_{a b}, \bar{Q}_{b a}\right\}}{n+m}$. Hence, $\frac{n}{n+m} \phi(p)+\frac{m}{n+m} \phi(q)=\frac{\sum_{a b \in A \times A} w(a b) \times \min \left\{\bar{P}_{a b}, \bar{P}_{b a}\right\}}{n+m}+$ $\frac{\sum_{a b \in A \times A} w(a b) \times \min \left\{\bar{Q}_{a b}, \bar{Q}_{b a}\right\}}{n+m}$. by $(\star)$, this is equal to $\sum_{a b \in A \times A} \frac{w(a b) \times \min \left\{\bar{Q}_{a b}+\bar{P}_{a b}, \bar{Q}_{b a}+\bar{P}_{b a}\right\}}{m+n}$.

This proves that the additivity constraint is always satisfied.

\subsection{Function 3}

Three agents First, we will broaden function 3 to three agents.

For three agents, there are at most $4^{3}=64$ matrices of pairwise comparisons possible ( in fact, there are less matrices). One can divide these matrices into four groups, related to the number of zeros on the upper and lower triangular:

In group 1, $\left\{\bar{P}_{a b}, \bar{P}_{b a}\right\}=\{0,3\}$ for three pairs $a b \in A \times A, a \neq b$, and $\left\{\bar{P}_{a b}, \bar{P}_{b a}\right\}=\{3,0\}$ for the other three pairs $a b \in A \times A, a \neq b$;

In group 2, $\left\{\bar{P}_{a b}, \bar{P}_{b a}\right\}=\{0,3\}$ for two pairs $a b \in A \times A, a \neq b,\left\{\bar{P}_{a b}, \bar{P}_{b a}\right\}=\{3,0\}$ for two pairs $a b \in A \times A, a \neq b$, for one pair $a b \in A \times A, a \neq b,\left\{\bar{P}_{a b}, \bar{P}_{b a}\right\}=\{2,1\}$, and for the last pair $a b \in A \times A, a \neq b,\left\{\bar{P}_{a b}, \bar{P}_{b a}\right\}=\{1,2\}$.

In group 3, $\left\{\bar{P}_{a b}, \bar{P}_{b a}\right\}=\{0,3\}$ for one pair $a b \in A \times A, a \neq b,\left\{\bar{P}_{a b}, \bar{P}_{b a}\right\}=\{3,0\}$ for one pair $a b \in A \times A, a \neq b$, while $\left\{\bar{P}_{a b}, \bar{P}_{b a}\right\}=\{2,1\}$ for two pairs $a b \in A \times A, a \neq b$, and $\left\{\bar{P}_{a b}, \bar{P}_{b a}\right\}=\{1,2\}$ for the last two pairs $a b \in A \times A, a \neq b$;

In group $4,\left\{\bar{P}_{a b}, \bar{P}_{b a}\right\}=\{2,1\}$ for three pairs $a b \in A \times A, a \neq b$, while for the other three pairs $a b \in A \times A, a \neq b,\left\{\bar{P}_{a b}, \bar{P}_{b a}\right\}=\{0,3\}$.

Each agent can have six different orders. This means that in total $6^{3}=216$ combinations of orders can be made.

In group 1, all situations where the three agents have the same order are captured (for example, all agents have preference $(\mathrm{a}, \mathrm{b}, \mathrm{c})$; then $\bar{P}=\left[\begin{array}{lll}0 & 3 & 3 \\ 0 & 0 & 3 \\ 0 & 0 & 0\end{array}\right]$ ) In all these cases, $\phi=0$; 


\section{Polarisation measurement on three alternatives}

An example of a situation that results in a matrix from group 2 is the following: Agent 1 has order $(a, b, c)$, agent 2 has order $(a, c, b)$ and agent 3 has order $(a, c, b)$ as well. Then $\bar{P}=$ $\left[\begin{array}{lll}0 & 3 & 3 \\ 0 & 0 & 1 \\ 0 & 2 & 0\end{array}\right]$. In this group, the orders of the different agents are still relatively close to each other; This is in correspondence with the outcome for $\phi$ for all these matrices: $\sum_{\forall 1 \leq i<j \leq m}\left(\bar{p}_{a b} \times \bar{p}_{b a}\right)=2$; hence $\frac{\sum_{\forall 1 \leq i<j \leq m}\left(\bar{p}_{a b} \times \bar{p}_{b a}\right)}{\frac{3}{4}\left(n^{2}-1\right)}=\frac{1}{3}$.

A situation that results in a matrix from group 3 is for example: agent 1 has order $(a, b, c)$, agent 2 has order $(\mathrm{a}, \mathrm{c}, \mathrm{b})$ and agent 3 has order $(\mathrm{c}, \mathrm{a}, \mathrm{b})$. Then $\bar{P}=\left[\begin{array}{lll}0 & 3 & 2 \\ 0 & 0 & 2 \\ 1 & 1 & 0\end{array}\right] . \quad \sum_{\forall 1 \leq i<j \leq m}\left(\bar{p}_{a b} \times \bar{p}_{b a}\right)=$ 4 , and therefore $\frac{\sum_{1 \leq i<j \leq m}\left(\bar{p}_{a b} \times \bar{p}_{b a}\right)}{\frac{3}{4}\left(n^{2}-1\right)}=\frac{2}{3}$ for all these matrices. All the combinations of orders that result into this polarisation measurement are relatively far spread.

Lastly, an example for a matrix from group 4: agent 1 has order $(a, b, c)$, agent 2 has order $(\mathrm{c}, \mathrm{a}, \mathrm{b})$ and agent 3 has order $(\mathrm{b}, \mathrm{c}, \mathrm{a})$. Then $\bar{P}=\left[\begin{array}{lll}0 & 2 & 1 \\ 1 & 0 & 2 \\ 2 & 1 & 0\end{array}\right]$. For all matrices in this group, $\sum_{\forall 1 \leq i<j \leq m}\left(\bar{p}_{a b} \times \bar{p}_{b a}\right)=6$, and therefore $\frac{\sum_{\forall 1 \leq i<j \leq m}\left(\bar{p}_{a b} \times \bar{p}_{b a}\right)}{\frac{3}{4}\left(n^{2}-1\right)}=1$. This is the highest score that can be obtained by three agents.

This means that for three agents, regularity is satisfied;

Moreover, neutrality is also satisfied, since combinations of orders will lead to the same matrices, as long as the 'distances' between the different pairs are the same.

By construction of this function, different profiles that lead to the same matrix of pairwise comparisons will always have the same measure of polarisation. Therefore, pairwiseness is always satisfied;

We leave the additivity constraint in the case of three agents for what it is. Instead, we will prove that it is not satisfied for more than three agents.

More than three agents For more than three agents, regularity is always satisfied; $\phi$ is always zero when all agents have the same preferred order, since in each pairwise multiplication, one of the entries is always zero. This means that the sum of these multiplications is always 


\section{Polarisation measurement on three alternatives}

zero, hence their sum is also always zero. The denominator is defined in such a way that it is always equal to the maximum value the numerator can get. Hence, $\phi$ is at most 1 .

Moreover, for the same reasons as that they were satisfied for three agents, neutrality and pairwiseness are also satisfied for any number of agents;

However, additivity is not always satisfied. To prove this, we consider four agents.

For four agents, 10 different outcomes for $\phi$ are possible. This is because $\left(\bar{p}_{a b} \times \bar{p}_{b a}\right)$ can be 0 , 3 and 4 . Since this holds for all three pairs of entries in the upper triangular and the reversed entries in the lower triangular, $\sum_{\forall 1 \leq i<j \leq m}\left(\bar{p}_{a b} \times \bar{p}_{b a}\right)$ can be $0,3,4,6,7,8,9,10,11$,and 12. Hence, $\phi$ can be $0, \frac{1}{4}, \frac{1}{3}, \frac{1}{2}, \frac{7}{12}, \frac{2}{3}, \frac{3}{4}, \frac{5}{6}, \frac{11}{12}$ and 1 .

To prove that the additivity constraint is not satisfied anymore for four agents, we consider a counterexample:

proof Consider the following situation: we have four agents: agent 1 has preference $(a, b, c)$, agent 2 has preference $(a, b, c)$, agent 3 has preference $(a, c, b)$ and agent 4 has preference $(c, a, b)$. Agent 1 and 2 together form profile $p$, and agent 3 and 4 together form profile $q$. Then, profile $p$ creates the following matrix: $\bar{P}=\left[\begin{array}{ccc}0 & 2 & 2 \\ 0 & 0 & 2 \\ 0 & 0 & 0\end{array}\right]$. According to our formula, $\phi=0$ for this profile. For profile q, we get this matrix: $\bar{P}=\left[\begin{array}{lll}0 & 2 & 1 \\ 0 & 0 & 1 \\ 1 & 1 & 0\end{array}\right]$. Here, $\phi=\frac{2}{3}$. Since these two matrices are pairwise conflict free, the additivity constraint requires that $\frac{1}{2} \phi(p)+\frac{1}{2} \phi(q)=\phi(p, q)$. Thus, $\frac{1}{2} \times 0+\frac{1}{2} \times \frac{2}{3}=\frac{1}{3}$. However, if agent $1,2,3$ and 4 are together in one profile, we get $\bar{P}=\left[\begin{array}{ccc}0 & 4 & 3 \\ 0 & 0 & 2 \\ 1 & 2 & 0\end{array}\right]$. This gives $\sum_{\forall 1 \leq i<j \leq m}\left(\bar{p}_{a b} \times \bar{p}_{b a}\right)=7$ and $\frac{\sum_{1 \leq i<j \leq m}\left(\bar{p}_{a b} \times \bar{p}_{b a}\right)}{\frac{3}{4}\left(n^{2}-1\right)}=\frac{7}{12}$. This contradicts additivity.

When the additivity constraint is not satisfied for four agents, this means that it is never satisfied for more than 6 agents, because we can always find the same counter example: The four agents from the previous example together form profile $p$, and the number of $m>2$ extra agents together form profile q. Moreover, all agents in profile $q$ have preferred order $(a, b, c)$. Then 


\section{Polarisation measurement on three alternatives}

$\bar{P}=\left[\begin{array}{ccc}0 & 4 & 3 \\ 0 & 0 & 2 \\ 1 & 2 & 0\end{array}\right], \bar{Q}=\left[\begin{array}{ccc}0 & m & m \\ 0 & 0 & m \\ 0 & 0 & 0\end{array}\right]$ and $\bar{P}+\bar{Q}=\left[\begin{array}{ccc}0 & 4+m & 3+m \\ 0 & 0 & 2+m \\ 1 & 2 & 0\end{array}\right]$. Then the following should hold: $\frac{4}{4+m} \times \frac{7}{12}+\frac{m}{4+m} \times 0=\frac{(3+m)+2(2+m)}{3 \times\left(\frac{1}{2}(m+4)\right)^{2}} \cdot \frac{(3+m)+2(2+m)}{3 \times\left(\frac{1}{2}(m+4)\right)^{2}}=\frac{7+3 m}{3 \times\left(\frac{1}{2}(m+4)^{2}\right)}=\frac{7+3 m}{\frac{1}{4} m^{2}+2 m+3.75}$. $\frac{7}{12+4 x}=\frac{7+3 m}{\frac{1}{4} m^{2}+2 m+3.75}$ when $m=-1.87805$, hence for $\mathrm{m}>6$, this can never be the case. This proves that the additivity constraint is not satisfied for any number of agents larger than three. Therefore, to prove that the additivity constraint is not satisfied for more than three agents, we only still have to prove that it is not satisfied for five agents. We prove this by the following counter example:

proof Consider the following situation: There are in total 5 agents, agent 1 up to 5 . Agent 1,2 and 3 all have preferred order $(a, b, c)$. Agent 4 has preferred order $(a, c, b)$ and agent 5 has preferred order $(c, a, b)$. Agent 1,2 and 4 together form profile $p$, and agent 3 and 5 together form profile $q$. Then $\bar{P}=\left[\begin{array}{lll}0 & 3 & 3 \\ 0 & 0 & 2 \\ 0 & 1 & 0\end{array}\right], \bar{Q}=\left[\begin{array}{ccc}0 & 2 & 1 \\ 0 & 0 & 1 \\ 1 & 1 & 0\end{array}\right]$ and $\bar{P}+\bar{Q}=\left[\begin{array}{ccc}0 & 5 & 4 \\ 0 & 0 & 4 \\ 1 & 1 & 0\end{array}\right]$. These two matrices are both pairwise conflict free. This means that the following should hold: $\frac{3}{5} \phi(p)+\frac{2}{5} \phi(q)=\phi(p, q) . \phi(p)=\frac{1}{3}$ and $\phi(q)=\frac{2}{3}$, thus $\phi(p, q)$ should be equal to $\frac{3}{5} \times \frac{1}{3}+\frac{2}{5} \times \frac{2}{3}=\frac{9}{15}$. However, $\phi(p, q)=\frac{4}{9}$. This contradicts the additivity constraint.

This completes the proof that the additivity constraint is not satisfied for more than three agents.

\section{Conclusion}

In this thesis, we searched for different polarisation expressing measures. We found three different functions. Function 1 was a first try-out. This function works for two agents, but cannot be applied to more than two agents. Function 2 is based on the symmetric differences of two different sets. This concept works perfectly for two agents. For more than two agents, this function had to be changed slightly to satisfy the additivity constraint. When the symmetric difference of two sets is replaced by the minimum of two corresponding entries, the function satisfies all the constraints. The third function was based on the principle matrix of pairwise comparisons. For two and three agents, this function works fine for all the constraints. For four agents or more, this function does not satisfy the additivity constraint anymore. Although this function does not satisfy the additivity constraint anymore, this function still generates reasonable 


\section{Polarisation measurement on three alternatives}

results that can be seen as an indication of the polarisation in a particular situation.

\section{Suggestions for further research}

In this thesis, we restricted ourselves to only three alternatives. But function two and three probably will work as well for more than three alternatives. The expansion of function two and three to more than three alternatives could therefore be an interesting topic for future research. Moreover, we discovered throughout this thesis, that the additivity constraint was the most restricting constraint. It was very difficult to find function that satisfied this constraint. If this constraint is removed, or formalised less restricting, there might be new opportunities for our three or other functions to be explored. Especially our function three would work perfect if the additivity constraint would be ignored. This can also be interesting for further research. It might also be interesting to pick up the concept of 'high value' swaps and a 'low value' swaps again. We discussed this topic very briefly when we were defining function 2 , and we concluded that this concept could not be used in this function, but it is possible that it can be applied on other functions. Lastly, future researchers could maybe try to capture the location of a particular alternative in the preferred order of an agent. Take for example the following situation: We have a profile $p$ with three agents, agent 1,2 and 3 , and 3 alternatives, $a, b$ and $c$. Agent 1 has preferred order $(a, b, c)$, agent 2 has preferred order $(a, b, c)$ and agent 3 has preferred order (b,c,a). Then function 2 gives $\phi_{2}(p)=\frac{4}{9}$ and function 3 gives $\phi_{3}(p)=\frac{2}{3}$. These functions indicate how difficult it is to make a compromise. But they give no information about which alternative could be the best compromise. In this case, since two of the three agents have alternative a as top preference, a logical conclusion seem that activity a will be picked. But agent 3 has this alternative indicated as least preferred option. Alternative $b$ might be a better option. This idea, where one does not only check whether a certain alternative is preferred over another alternative or not, but also check in some way how much a certain alternative is preferred over another, is not captured in this thesis. However, we think that this is a very interesting concept for future research. 
Polarisation measurement on three alternatives

\section{References}

[1] Baldiga, K. A. and Green, J. R. (2013). Assent-maximizing social choice. Social Choice and Welfare, 40(2):439-460.

[2] Duclos, J.-Y., Esteban, J., and Ray, D. (2004). Polarization: concepts, measurement, estimation. Econometrica, 72(6):1737-1772.

[3] Esteban, J.-M. and Ray, D. (1994). On the measurement of polarization. Econometrica, 62:819-819.

[4] Perloff, J.M. (2012) Microeconomics. University of Berkeley, California. A4-A8. 